\title{
Theoretical motivation for electroweak physics with neutrinos
}

\author{
ANDRÉ DE GOUVÊA* \\ Northwestern University \\ E-mail: degouvea@northwestern.edu
}

Here I present a short overview of the physics reach of next-generation neutrino fixed-target experiments, concentrating on improving our understanding of electroweak interactions and looking for new physics at the TeV-scale. In particular, I draw attention to precision measurements of neutrino electron (pseudo)elastic scattering, $v_{\mu}+e^{-} \rightarrow v_{\mu,(\text { other })}+e^{-}$.

10th International Workshop on Neutrino Factories, Super beams and Beta beams

June 30 - July 52008

Valencia, Spain

${ }^{*}$ Speaker.

$\dagger$ It is a pleasure to thank the Local Organizing Committee for putting together an excellent program in a very relaxing and inspiring venue. I also thank Kevin McFarland for the invitation to present this talk in the Neutrino Scattering Physics Working Group. This work is sponsored in part by the US Department of Energy Contract DE-FG0291ER40684. 
Introduction: Since the early 1960's [1], neutrino-matter scattering experiments have provided indispensable information concerning both the internal structure of nuclei and nucleons and the nature of weak interactions. The latter is particularly true due to the fact that neutrinos, unlike electrons, do not participate in electromagnetic interactions, allowing one to look at purely weakinteracting processes. Finally, neutrino-matter scattering provides a direct window toward both charged-current and neutral-current weak processes.

It is widely anticipated that, in the near future, intense neutrino sources of all energies and different flavors will be available (for a brief overview presented in this conference, see [2]). These are required in order to pursue long-baseline neutrino oscillation experiments, the primary driving force of the NuFact conference series. Once an intense proton beam is available, the properly designed "near detector" serves as an excellent laboratory of neutrino-matter scattering. Recently, the possibility of utilizing the soon-to-become-available Tevatron at Fermilab as a proton source for a high energy neutrino fixed target experiment $-\mathrm{NuSOnG}-$ has been considered in detail $[3,4]$.

In this talk, I briefly discuss the ability of next generation neutrino-matter scattering experiments to test the standard model of electroweak interactions and search for new TeV-scale physics. I'll concentrate on results obtained assuming the NuSOnG setup [3] and point readers to, for example, $[2,5,6,7]$ for more detailed discussions involving other neutrino sources. As is well known, neutrino-matter scattering also serves as a unique laboratory for understanding the structure of nuclei and nucleons. Some results along this line were presented in this meeting ([2, 4, 7]) and will not be discussed here. For more details regarding the reach of a neutrino factory beam see, for example, [5].

Neutrino-Charged Fermion Scattering As Probe of New Physics: For center of mass energies well below the $W$-boson and $Z$-boson masses (guaranteed to be true in any fixed target experiment one can envision doing in the foreseeable future) neutrino scattering on a fermion $f$ $(f=e, u, d)$ is govern by the following Lagrangian:

$$
\mathscr{L}=-2 \sqrt{2} G_{F}\left(g_{L}^{v} \bar{v}_{L} \gamma_{\mu} v_{L}\right)\left[g_{L}^{f} \bar{f}_{L} \gamma^{\mu} f_{L}+g_{R}^{f} \bar{f}_{R} \gamma^{\mu} f_{R}\right],
$$

where $G_{F}$ is the Fermi constant, $g_{L, R}$ are dimensionless couplings and the subscripts $L$ and $R$ refer to the chirality of the fermion field. Eq. (1) assumes that the neutrino beam is $100 \%$ left-chiral. While this is not strictly correct once neutrino masses are taken into account, right-chiral "contaminations" are negligible due to the smallness of the neutrino masses and the left-handed nature of the weak interactions. In the standard model, for $v \neq v_{e}$,

$$
\begin{aligned}
& g_{L}^{v}=\sqrt{\rho}\left(+\frac{1}{2}\right), \\
& g_{L}^{f}=\sqrt{\rho}\left(I_{3}^{f}-Q^{f} \sin ^{2} \theta_{W}\right), \\
& g_{R}^{f}=\sqrt{\rho}\left(-Q^{f} \sin ^{2} \theta_{W}\right),
\end{aligned}
$$

Where $Q$ is the electric charge and $I_{3}$ the field's third component of weak isospin. At tree-level, $\rho=1$. Loop corrections affect both $\rho$ and the definition of $\sin ^{2} \theta_{W}$.

While the results of neutrino-matter scattering experiments are often translated into measurements of the Weinberg angle $\theta_{W}$, it is clear that, in principle, one can measure two independent couplings: $g_{L}^{v} g_{L}^{f}$ and $g_{L}^{v} g_{R}^{f}$. The current status of such neutrino measurements is summarized in [3]. 
Here I'll concentrate on the physics of neutrino-electron scattering, for several reasons. Most precision measurements of neutrino-matter scattering concentrated on neutrino-quark scattering. The reason for this is that the total cross-section for neutrino-quark scattering is much larger than that for neutrino-electron scattering, and isolating neutrino-electron scattering events requires a detector designed for that purpose. The last dedicated experiment for measuring neutrinoelectron scattering was the CHARM II experiment which accumulated less than 6,000 neutrino and antineutrino-electron scattering events combined [8]. A next-generation experiment like NuSOnG would be capable of accumulating up to 75,000 events. Neutrino-electron scattering is, arguably, the cleanest purely neutral-current process (unlike neutrino-quark scattering, which has to be extracted from neutrino-nucleus scattering). Indeed, it is included among the first processes calculated once the standard model was shown to be renormalizable [9]. Finally, the most precise measurement of neutrino-quark scattering revealed a discrepancy with respect to simplest standard model expectations [10]. One way to check this so-called NuTeV anomaly is to measure the neutrino neutral current couplings in other channels, especially neutrino-electron elastic scattering.

It is important to mention that in order to take advantage of the neutrino-electron data samples, one must understand very well the neutrino beam. This can be done in a variety of ways. In a neutrino factory, for example, the neutrino beam energy spectrum is well understood, along with its normalization (see, for example, [11]). In the case of a pion-decay neutrino beam, one needs to resort to the measurement of ratios in order to reduce uncertainties due to the normalization and energy spectrum of the neutrino beam. In NuSOnG a very clean measurement consists of the ratio of neutrino-electron scattering events to inverse muon decay: $v_{\mu}+e^{-} \rightarrow v_{e}+\mu^{-}$. This can only be achieved for (a) muon neutrinos (as opposed to other flavors or muon antineutrinos) and (b) high energy neutrinos $\left(E_{v}>10.9 \mathrm{GeV}\right)$.

New "heavy" physics $\left(M_{\text {new }} \gg \sqrt{s}\right)$ modifies the coefficients $g_{L}^{v} g_{L}^{f}$ and $g_{L}^{v} g_{R}^{f}$ from their standard model values assuming one can ignore new physics effects that couple to a scalar or pseudoscalar neutrino current $\bar{v}_{R} v_{L}$ or $\bar{v}_{R} \gamma_{5} v_{L}$ (or the equivalent for Majorana neutrinos). This is a good assumption given that these contributions are suppressed due to the smallness of the neutrino (and first generation charge fermion) masses. It is convenient to parameterize the new physics via

$$
\mathscr{L}_{\mathrm{NSI}}^{e}=+\frac{\sqrt{2}}{\Lambda^{2}}\left[\bar{v}_{\alpha} \gamma_{\sigma} P_{L} v_{\mu}\right]\left[\cos \theta \bar{e}_{L} \gamma^{\sigma} e_{L}+\sin \theta \bar{e}_{R} \gamma^{\sigma} e_{R}\right]
$$

where $\Lambda$ indicates the scale of new physics and $\theta$ characterizes both the "handedness" of the new physics couplings and whether it interferes constructively or destructively with the standard model contribution. $\alpha=\mu$ or $\alpha \neq \mu$ indicates the flavor of the outgoing neutrino. In the $\alpha=\mu$ case, the new physics contribution interferes with the standard model one, while in the $\alpha \neq \mu$ case (which is referred to as pseudo-elastic scattering) the standard model mediated scattering is is not coherent with the new physics driven contribution.

Fig. 1 depicts the sensitivity of NuSOnG to the new physics scale $\Lambda$ as a function of $\theta$. Independent of $\theta$, NuSOnG is sensitive to $\Lambda \lesssim 3.5 \mathrm{TeV}$ for $\alpha=\mu$ and $\Lambda \lesssim 1.2 \mathrm{TeV}$ for $\alpha \neq \mu$. The new physics reach of NuSOnG is competitive with other leptonic probes (which involve only charged leptons), including LEP2 [12], and precision measurements of Møller scattering [13].

If new physics is discovered in neutrino-electron scattering, other experimental data will be required in order to help sort out its nature. More information can be provided by other probes of new 


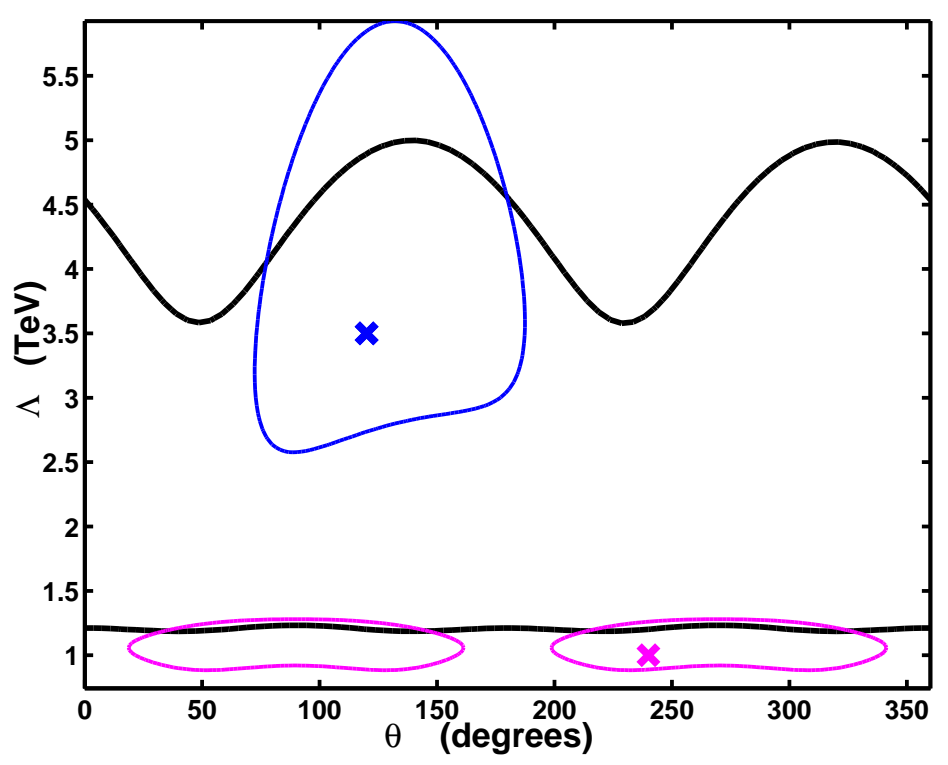

Figure 1: (DARK LINES) 95\% confidence level sensitivity of NuSOnG to new heavy physics described by Eq. (5) when $\alpha=\mu$ (higher curve) and $\alpha \neq \mu$ (lower curve). (CLOSED CONTOURS) NuSOnG measurement of $\Lambda$ and $\theta$, at the $95 \%$ level, assuming $\alpha=\mu, \Lambda=3.5 \mathrm{TeV}$ and $\theta=2 \pi / 3$ (higher, solid contour) and $\alpha \neq \mu, \Lambda=1 \mathrm{TeV}$ and $\theta=4 \pi / 3$ (lower, dashed contour). Note that in the pseudoelastic scattering case $\left(v_{\alpha} \neq v_{\mu}\right) \theta$ and $\pi+\theta$ are physically indistinguishable.

physics at the $\mathrm{TeV}$ scale including the LHC experiments and precision measurements of neutrinoquark scattering (which will also be performed at NuSOnG and all other neutrino-matter scattering experiments). Different new physics scenarios one is sensitive through neutrino-matter scattering will manifest themselves in different ways. Some affect all electroweak precision observables (involving quarks, charged leptons, etc) in the same way. Others affect only the neutrino neutral and weak currents, while a different subset affects only neutrino-quark scattering or neutrino-charged lepton scattering. Several specific new physics scenarios can be probed by a high statistics, high precision measurement of neutrino-matter interactions. NuSOnG's reach to several heavy new physics scenarios is discussed in [3] and references therein.

Other neutrino sources also provide an excellent window into the weak interactions and new TeV-scale physics. Some are discussed in detail in [2, 5, 6, 7]. Table 1 from [6] provides a flavor of the different searches and measurements one should be able to perform.

Summary and Concluding Remarks: An experiment capable of precisely measuring neutrinomatter scattering (especially neutrino-electron scattering) is sensitive to new physics at the $\mathrm{TeV}$ scale. The sensitivity of such experiments is complementary to the LHC, as follows. If the LHC finds evidence for physics beyond the standard model, a precision neutrino scattering experiment will help elucidate the nature of this new physics. Should the LHC confirm the standard model, a neutrino scattering experiment is sensitive to new $\mathrm{TeV}$ scale physics that can easily evade LHCrelated probes.

A comprehensive neutrino scattering experiment further provides the only definitive means for understanding the NuTeV anomaly, regardless of whether it is due to new physics in the electroweak 
Table 1: Results on the precision (68\% confidence) of parameter extraction, assuming a 100 ton detector located $100 \mathrm{~m}$ from the neutrino source. The bounds in parenthesis are computed assuming a more conservative systematic uncertainty on the neutrino flux. See [6] for the relevant details

\begin{tabular}{|c||c|c|c|c|}
\hline Neutrino Source & $\sin ^{2} \theta_{W}$ & magnetic moment & $Z^{\prime}$ coupling $\varepsilon$ & $\rho$ \\
& $\%$ & $68 \%$ & $68 \%$ & $\%$ \\
\hline Reactor & 0.82 & $4.8 \times 10^{-10} \mu_{B}$ & $2.0 \times 10^{-3}$ & 1.1 \\
$\mu^{-} v$-factory & $0.04(8.62)$ & $3.1(12.4) \times 10^{-11} \mu_{B}$ & $3.3(8.7) \times 10^{-4}$ & $0.06(0.93)$ \\
$\beta$-beam $v_{e}\left({ }^{18} \mathrm{Ne},\right)$ & $0.34(7.60)$ & $3.0(6.6) \times 10^{-10} \mu_{B}$ & $9.8(16.3) \times 10^{-4}$ & $0.39(2.4)$ \\
$\beta$-beam $\bar{v}_{e}\left({ }^{6} \mathrm{He}\right)$ & $0.22(5.72)$ & $2.6(6.7) \times 10^{-10} \mu_{B}$ & $7.7(14.2) \times 10^{-4}$ & $0.75(3.1)$ \\
$\pi$-decay super-beam & $0.48(9.92)$ & $1.8(6.6) \times 10^{-10} \mu_{B}$ & $2.7(6.4) \times 10^{-3}$ & $3.3(7.3)$ \\
\hline
\end{tabular}

sector or to "old physics" effects related to nuclear physics or the structure of nucleons.

Finally, even in the absence of new physics at the $\mathrm{TeV}$ scale, neutrino-matter scattering probes a different sector of the standard model (compared to the LHC, "flavor factories", etc) and contributes non-trivially to electroweak precision measurements and several otherwise unaccessible sectors of QCD.

\section{References}

[1] G. Danby, J. M. Gaillard, K. Goulianos, L. M. Lederman, N. B. Mistry, M. Schwartz and J. Steinberger, Phys. Rev. Lett. 9, 36 (1962).

[2] R. Petti, Neutrino Scattering at Future Oscillation Facilities, in proceedings of 10th International Workshop on Neutrino Factories, Super beams and Beta beams (NuFact08), June 30 - July 5 2008, Valencia, Spain POS (NUFACT08) 015.

[3] T. Adams et al. [NuSOnG Collaboration], arXiv:0803.0354 [hep-ph].

[4] I. Schienbein, Future Experiments: NuSOnG, in proceedings of 10th International Workshop on Neutrino Factories, Super beams and Beta beams (NuFact08), June 30 - July 5 2008, Valencia, Spain POS (NUFACT08) 068.

[5] M. L. Mangano et al., arXiv:hep-ph/0105155.

[6] A. de Gouvêa and J. Jenkins, Phys. Rev. D 74, 033004 (2006).

[7] R. Petti, Future Experiments: Project X, in proceedings of 10th International Workshop on Neutrino Factories, Super beams and Beta beams (NuFact08), June 30 - July 5 2008, Valencia, Spain POS (NUFACT08) 069.

[8] D. Geiregat et al. [CHARM-II Collaboration], Phys. Lett. B 259, 499 (1991).

[9] G. 't Hooft, Phys. Lett. B 37, 195 (1971).

[10] G. P. Zeller et al. [NuTeV Collaboration], Phys. Rev. Lett. 88, 091802 (2002) [Erratum-ibid. 90, 239902 (2003)].

[11] A. Bandyopadhyay et al. [ISS Physics Working Group], arXiv:0710.4947 [hep-ph].

[12] C. Amsler et al. [Particle Data Group], Phys. Lett. B 667, 1 (2008).

[13] P. L. Anthony et al. [SLAC E158 Collaboration], Phys. Rev. Lett. 95, 081601 (2005). 